\title{
Alphanumeric Character Recognition Based on BP Neural Network Classification and Combined Features
}

\author{
Yong Luo ${ }^{1}$, Shuwei Chen ${ }^{1}$, Xiaojuan $\mathrm{He}^{2}$, and Xue $\mathrm{Jia}^{1}$ \\ ${ }^{1}$ School of Electrical Engineering, Zhengzhou University, \\ Zhengzhou 450001, Henan, China \\ Email: luoyong@zzu.edu.cn; swchen@zzu.edu.cn; 365410642@qq.com \\ ${ }^{2}$ No.6 Institute of Project Planning \& Research of Machinery Industry Limited Company, \\ Zhengzhou 450007, Henan, China \\ Email: hexiaojuan456@163.com \\ Received 4 October 2012 \\ Accepted 9 March 2013
}

\begin{abstract}
This paper puts forward a new method of alphanumeric character recognition based on BP neural network classification and combined features. This method firstly establishes three BP networks respectively for three categories of characters which are classified according to their Euler numbers, with the combination of grid feature and projection feature as the input of each BP network. When recognizing a character, its combined features are fed into the three BP networks simultaneously without the necessity for judging its Euler number. The final recognition result is elaborated by synthetically analyzing the outputs of three BP networks. Experimental results show that the proposed method can effectively improve the recognition ability and efficiency, and has a good property of fault tolerance and robustness. Furthermore, the weight coefficients of combined features for each BP network are optimized, which can further improve the recognition rate.
\end{abstract}

Keywords: Character Recognition, Combined Features, BP Network Classification, Euler Number

\section{Introduction}

Alphanumeric characters recognition is a key research area of pattern recognition, and it has been widely applied in license plate number and library call number recognition. The success of pattern recognition, to a large extent, depends on the stability and accuracy of extracted features. The quality of the extracted features will directly affect the recognition result ${ }^{1}$. According to the unified entropy theory of pattern recognition, we must ensure that the extracted features contain sufficient information to get a higher recognition rate ${ }^{2}$.

A variety of methods have been developed for extracting a character features. For example, Bandara explored fuzzy feature descriptions for character feature recognition ${ }^{3}$. Plamondon put forward a way of using machine learning, such as hidden Markov model (HMM), to extract character features ${ }^{4}$. Kim developed a character feature extraction method by using support vector machine (SVM) ${ }^{5}$. Woods employed Sobel edge detector to analyze and classify image-based spam ${ }^{6}$. Tangent vectors are also widely used for character feature extraction ${ }^{7}$. Some combined recognition methods have also been proposed for character feature recognition, such as, Hassiba put forward an approach of combining neural network with SVM to recognize alphanumeric characters ${ }^{8}$. Camastra developed an approach of 
combining neural gas and learning vector quantization for cursive character recognition ${ }^{9}$.

In character recognition, alphanumeric features are generally divided into statistical features, direction features and structural features ${ }^{10}$. Traditional statistical features include stroke density feature, geometric moment feature, histogram feature, grid feature, feature points feature and so on. Statistical features have the characteristics of strong anti-interference, simple algorithm of matching and classification, weak subdivision and bad capability of distinguishing between similar characters. Direction features generally include chain code feature, gradient feature and peripheral direction contributivity (PDC) ${ }^{11}$. The universally used structural features include Euler number, skeleton feature, topological feature, concavity features, profile structure feature and so on ${ }^{12}$. The structural features have good compatibility with the changing of the character and capability of distinguish between similar characters, but if it cannot accurately obtain the structural feature, then recognition effect will be greatly affected. Therefore, along with the research on character recognition, the combination of two features is an efficacious method. For example, Thome and Vacavant proposed a way to get the topology and geometry-related features of the character by Reeb graph, and used the hierarchical neural networks to achieve recognition ${ }^{13}$. Lu put forward the idea of using Zernike rule to get the entire features, using the Wavelet transform to get the detailed features, and then classify and identify them by BP network, respectively ${ }^{14}$. One can also use subregion recognition firstly, and then apply an overall integrated approach to recognize a character. Such as the idea proposed by $\mathrm{Li}$ and Suen ${ }^{15}$ that "the easier part recognition is first carried out, then recognition of the entire pattern can be completed by integrating the results of part recognition”.

After feature extraction, in general, classifiers should be used to train and distinguish between characters. Liu sorted the classifiers as: MLP classifier, RBF classifier, LVQ classifier, DLQDF classifier and support vector machine classifier ${ }^{11}$. Vamvakas made a two-stage classification scheme based on the level of granularity features of handwriting character's subdivisions ${ }^{16}$. Pauplin presented dynamic Bayesian networks models trained for the classification of handwritten digit ${ }^{17}$.

This paper puts forward a new method for character recognition based on integration of two types of features. In order to ensure extracted features contain enough information of character, this paper chooses two groups of high dimension features to recognize character: grid feature and projection feature. Because they are essentially statistical features, not sufficient for structural description of character shape. This paper also uses structural feature of Euler number in order to overcome this problem.
Since the BP neural network has advantages of information distributed storage, parallel processing, self-organization, and self-learning, etc., it has been widely used in image pattern recognition ${ }^{8,13}$. Aiming at solving the current bottlenecks of low recognition rate and complex algorithm in image pattern recognition, this paper firstly roughly classifies all alphanumeric characters into three categories by structural feature of Euler number, and establishes three BP neural networks corresponding to each category. Each BP network is trained by the samples of corresponding category, respectively. The grid feature and projection feature of a character constitute its combined features, which serve as the input of each BP network. When recognizing a character, its combined features under the optimal weight coefficients are fed into the three BP networks simultaneously, without the necessity to select one of three BP networks according to judgment of its Euler number. A synthetic analysis of the outputs of the three BP networks is then applied to determine the final recognition result. The experimental results indicate that the method can effectively improve recognition ability and efficiency, and has a good property of fault tolerance and robustness.

\section{Single Feature}

\subsection{Structural feature}

Euler number reflects topology feature of image, which is equal to the number of total targets of image minus the number of holes of image. In the binary image, a target is a set of 4-connected or 8-connected points, whose values are 1 . The so-called 4- connected rule is that only horizontal and vertical pixels can be connected; 8 -connected rule is that all eight connections pixels can be connected as shown in Figure 1 and Figure 2. To denote the diagonal connecting pixels, the 8-connected rule only needs one value, while 4-connected rule takes two values, which makes computation complex and storage large, so this paper chooses the 8-connected rule utilized to the experiment study.

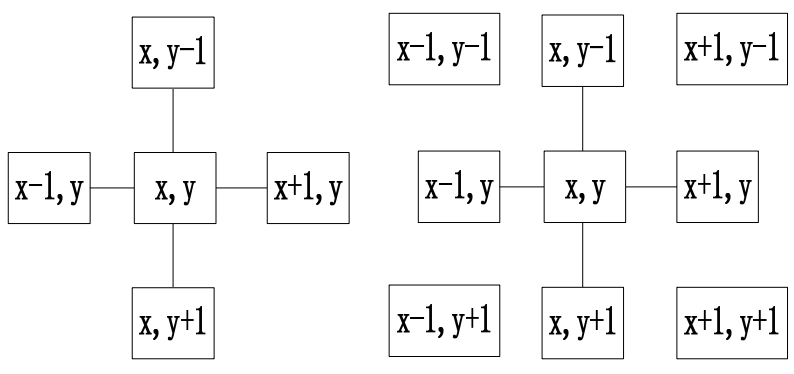

Fig. 1 4- connect

Fig. 2 8- connect 


$\begin{array}{llll}0 & 1 & 1 & 0 \\ 1 & 0 & 0 & 1 \\ 1 & 0 & 0 & 1 \\ 0 & 1 & 1 & 0 \\ 1 & 0 & 0 & 1 \\ 1 & 0 & 0 & 1 \\ 0 & 1 & 1 & 0\end{array}$

Fig. 3 Binary image matrix of “ 8 ”

Figure 3 shows the binary image matrix of "8". The image contains a target in accordance with the 8-connect rule, while the number of holes is 2 . So its Euler number is -1 .

26 letters and 10 digits are divided into three categories by their Euler numbers: $Q_{1}=\{8, B$ and $Q\}$, whose Euler numbers are $-1 ; \mathrm{Q}_{2}=\{0,4,6,9, \mathrm{~A}, \mathrm{D}, \mathrm{O}, \mathrm{P}$, $\mathrm{R}, \mathrm{W}\}$, whose Euler numbers are $0 ; \mathrm{Q}_{3}=\{1,2,3,5,7, \mathrm{C}$, E, F, G, H, I, J, K, L, M, N, S, T, U, V, X, Y, Z \}, whose Euler numbers are 1.

\subsection{Grid feature}

Binary image is divided into uniform grids, such as $2 \times 2$, $4 \times 4$, and the grid feature is constituted by the numbers of pixels in the grids, and the number is counted from left to right, top to bottom. For sample of $32 \times 16$ binary images, this paper selects grid of $4 \times 4$, so the dimension of grid feature is $32 \times 16 /(4 \times 4)=32$. In Figure 4 , the numbers of black pixels in the 32 regions of character “A” respectively are: [0 350088006100059037 11341012473781258 13]. This paper takes one grid as a unit, even if some pixels are distributed wrongly within the grid, the pixels' statistical values of whole grid region generally are the same. Therefore, the grid feature has a property of better anti-interference.

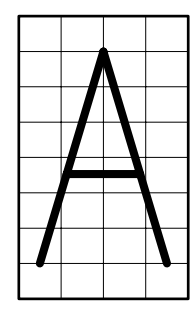

Fig. 4 Example of grid feature

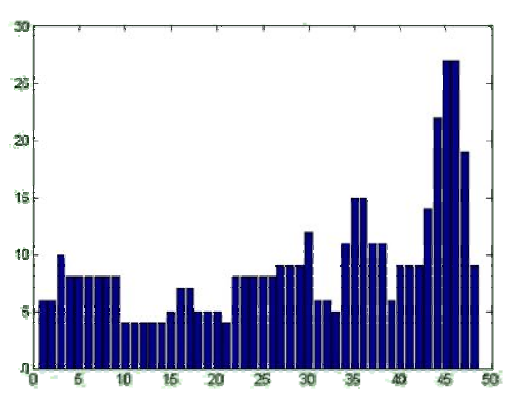

Fig. 5 Projection feature of " 3 "

\subsection{Projection feature}

Scanning from left to right, top to bottom, projection feature is the numbers of pixels, at which scanning line intersects with the binary image. For the sample of $32 \times$ 16 binary image, this paper obtains $32+16=48$ dimensional projection feature. Projection feature includes more information of image. Figure 5 shows the projection feature of “3”. (1-32) of abscissa in Fig. 5 indicate rows, through which scan line passes. Correspondingly, (33-48) of abscissa indicate columns, and the ordinate is the number of pixels at which scanning line intersects with a binary image.

\section{BP Recognition Classification Method}

Since all 36 alphanumeric characters can be divided into three categories $\mathrm{Q}_{1}, \mathrm{Q}_{2}, \mathrm{Q}_{3}$ by their Euler numbers, this paper can make use of the Euler numbers to coarsely classify all 36 alphanumeric characters, and then establish one BP network for each category. The input of each network is the combination of grid feature and projection feature of a character, and each network's correct output is a binary code corresponding to the character. After being trained by the samples of each category, the three trained BP network are saved. If a character is to be recognized, the combined features of the character are fed into three BP networks to obtain three results simultaneously. If the output of a BP network is a binary code corresponding to an element of the network's category, the recognition result of the network will be correct, otherwise it is wrong. IF one and only one recognition result of the three networks is correct, the character recognition is successful, otherwise the character recognition is wrong or incomplete.

The proposed method essentially establishes three BP networks according to the Euler numbers of all 36 alphanumeric characters. But there is no need to judge a character's Euler number. Because we recognize a character using three BP synchronously, and then make a comprehensive analysis of recognition results of three BP models to determine the final recognition result. Some existing works focus on recognizing a character using different BP networks according to its Euler number. In these existing works, the Euler number must be obtained before recognizing a character. Therefore, the proposed method is essentially different from that of choosing different BP network to recognize a character according to its Euler number (EBP). Since this paper does not use Euler number in actual recognition process, you can effectively avoid the error caused by the change of Euler number due to handwriting fracture. So the method has a good property of fault tolerance and robustness.

One BP network is trained by the training samples of its category. Therefore, the BP network has better adaptability and recognition rate for a character whose Euler number is the same as the BP network. Consequently the method can quickly recognize objects from different categories. In the same category, objects' combined features are fed into the BP neural network, and the recognition rate depends on the differences of combined features between objects. Moreover 
recognition accuracy depends on the generalization ability of BP network for the smaller differences of combined features between objects in the same category, such as " 1 " and "I".

For specific implementation, firstly this paper carries out the coarse classification by their Euler numbers to obtain three categories identified as $\mathrm{Q}_{1}, \mathrm{Q}_{2}$, $\mathrm{Q}_{3}$, and establishes one BP network for each category, which is net(-1), net(0) and net(1), respectively. The number in parenthesis represents the Euler number of the network corresponding to the category. Then we respectively train the network by samples of each category and save the trained network. Finally, the test samples are put into three networks, respectively, and the outputs of three networks are $\mathrm{Re}_{1}, \mathrm{Re}_{2}, \mathrm{Re}_{3}$. If $\mathrm{Re}_{\mathrm{i}}$ is a binary code corresponding to certain element of $\mathrm{Q}_{\mathrm{i}}$ category, then $\mathrm{Re}_{\mathrm{i}}$ is correct. Otherwise it is wrong. The recognition results of three networks are synthetically judged as the following:

If $\mathrm{Re}_{1}, \mathrm{Re}_{2}, \mathrm{Re}_{3}$ are all wrong, then the recognition result is wrong.

If one and only one of $\mathrm{Re}_{1}, \mathrm{Re}_{2}, \mathrm{Re}_{3}$ is correct, the recognition result is correct.

If two or all of $\mathrm{Re}_{1}, \mathrm{Re}_{2}, \mathrm{Re}_{3}$ are correct, the recognition result is incomplete, and the final right correct result must be one of them. Therefore, other features of the character, such as Euler number or geometric moment feature, should be used for further judgment.

The flow of recognition algorithm is shown in Figure 6.

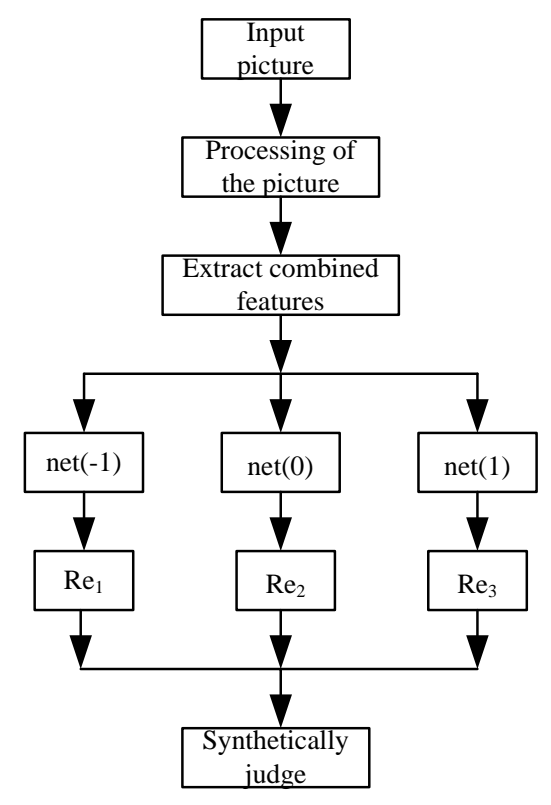

Fig. 6 The flow of recognition algorithm

Sometimes characters may be quite unclear in practice, which will lead to misjudgment of the Euler number. Since this paper only uses Euler number to classify all 36 alphanumeric characters, so as to establish BP network model for different categories, it is unnecessary to identify the Euler number of a character. Therefore, the recognition result of BP network is not affected by misjudgment of the Euler number due to the reason that Euler number is not the substantive judgment criterion for a character in the method.

Despite Euler number is not a key factor in the method, we can use it to pre-process an alphanumeric character. Since the Euler number of alphanumeric characters can only be $-1,0$ or 1 , it would be incorrect if an Euler number is judged as the other numbers, such as 2 or -2 . In this case, we need to do more image processing for re-identification. Moreover, if all the results of three BP outputs are correct, Euler number can be used to analyze the final result.

\section{Combined Features}

This paper combines the grid feature and the projection feature according to a certain weight coefficients to constitute a combined features vector. Given grid feature is $X_{1 \times 32}$, and projection feature is $Y_{1 \times 48}$, and the weight coefficients of them are $w_{1}$ and $w_{2}$, which all belong to [0, $1]$, and $w_{1}+w_{2}=1$. The combined features can be expressed as:

$$
V_{\text {comb }}=\left[w_{1} X_{1 \times 32}, w_{2} Y_{1 \times 48}\right]
$$

In order to obtain the optimal combination weight coefficients, this paper defines the feature weight vector: $\mathrm{w}=[\mathrm{w} 1, \mathrm{w} 2]$ and equally divides the straight line of $\mathrm{w} 1$ and w2 in the interval of 0.125 as shown in Fig. 7. One point on the line represents a combination of grid feature and projection feature with different weight coefficients. Recognition rates of the system are different under different weight coefficients of two features. In this paper, the optimal weight coefficients of combined features of each BP network are determined by experiments respectively, and Section 6.2 shows the experimental results.

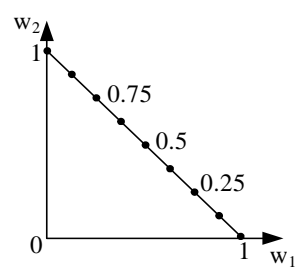

Fig. 7 The choice of weight coefficients

\section{Structure Design of BP Network}

Theoretical analysis showed that feed forward networks with a single hidden layer can map all continuous functions ${ }^{18}$. So this paper designs a single hidden layer BP network. 
1) The number of input layer neurons is the feature vector $V_{\text {comb }}$ dimension, that is, 80 .

2) The number of output layer neurons is 6 . In this paper, the $0 \sim 9$ and $\mathrm{A} \sim \mathrm{Z}$ are encoded by six binary numbers. The desired output vectors of 36-character codes are shown in Table 1. Since the activation function of neuron is a s-type function, so the desired interval of output is $(0,1)$, but cannot be 0 or 1 . Being 0 or 1 may cause the algorithm not converge. Therefore, this algorithm uses 0.1 to represent 0 , and 0.9 to represent 1 .

Table 1 Character encoding

\begin{tabular}{|c|c|c|}
\hline $\begin{array}{c}\text { 0 encoding: } \\
(0.1,0.1,0.1,0.1,0.1,0.1)\end{array}$ & $\cdots$ & $\begin{array}{c}\text { 9 encoding: } \\
(0.1,0.1,0.9,0.1,0.1,0.9)\end{array}$ \\
\hline $\begin{array}{c}\text { A encoding: } \\
(0.1,0.1,0.9,0.1,0.9,0.1)\end{array}$ & $\cdots$ & $\begin{array}{c}\text { Z encoding: } \\
(0.9,0.1,0.1,0.1,0.9,0.9)\end{array}$ \\
\hline
\end{tabular}

For net(-1) corresponding to $\mathrm{Q}_{1}$ category, the ideal outputs: "8” encoding for $(0.1,0.1,0.9,0.1,0.1,0.1)$, “ $B$ " encoding for $(0.1,0.1,0.9,0.1,0.9,0.9)$, “Q” encoding for $(0.1,0.9,0.9,0.1,0.9,0.1)$. The ideal outputs of net $(0)$ and net (1) are similar to this.

3) Hidden layer nodes are determined according to an empirical formula. Maren held that the number of the hidden layer nodes can be decided by the formula $\sqrt{p \times q}$ if $p>q$, and $p, q$ respectively represents the number of input and output nodes ${ }^{17}$. The number of hidden nodes is set to be $\sqrt{80 \times 6} \approx 22$ in this paper.

In summary, the structures of three BP network are all 80-22-6.

\section{Experiment}

\subsection{Sample collection}

The pictures of alphanumeric characters whose fonts are Times New Roman and whose sizes are 20pt, including normal, italic, bold, italic bold, respectively, one for each character, are generated from the drawing board. The rest of the samples are made using Matlab to add 'salt \& pepper', 'gaussian' and other noises to each clear sample, which are shown in Figure 8. A total of 40 sample pictures are generated for each character. So there are a total of 1440 sample pictures for 36 characters. 80\% sample pictures of each character are extracted for the training samples, and the rest of $20 \%$ are the test samples. Since the $\mathrm{Q}_{1}$ category has three characters, the total number of training samples of net $(-1)$ is $3 \times 40 \times 80 \%=$ 96. In the same way, the number of training samples of net(0) and net(1) is 320 and 736, respectively. The number of test samples of net(-1) is $3 \times 40 \times 20 \%=24$. Likewise, that of net(0) and net(1) is 80 and 184, respectively. The recognition rates of three networks are calculated by the following formula:

$$
P_{i}=T_{i} / M_{i}
$$

where $P_{i}$ represents recognition rate of one network; $T_{i}$ represents the number of correctly recognized samples of the network; $M_{i}$ represents the total number of test samples of the network.

\section{8}

Fig. 8 Example of sample pictures

\subsection{Experimental results}

This paper got the optimal weight coefficients of combined features for all BP networks based on the points of Figure 7 . The combined features under different weight coefficients are fed into one neural network to obtain different recognition rate. We selected the weight coefficients corresponding to the highest recognition rate as the optimal weight coefficients of the network.

Since only three elements in $\mathrm{Q}_{1}$, the recognition rates of net (-1) under different weight coefficients are all high. If $w_{1}=0.5$ and $w_{2}=0.5$, the recognition rate of net $(-1)$ achieved $100 \%$ by experiment. $Q_{2}$ has 10 elements, so the recognition rates of net(0) are slightly different under different weight coefficients. If $w_{1}=0.625$ and $w_{2}$ $=0.375$, the recognition rate of net $(0)$ achieved $95 \%$ by experiment. Among them, the number " 0 " is often recognized as “ $\mathrm{O}$ ”, which is a dominating factor causing the decline of recognition rate. $\mathrm{Q}_{3}$ altogether contains 23 element, and the recognition rates of net(1) are shown in Table 2 under different weight coefficients. Experimental results showed that if $w_{1}=0.75, w_{2}=0.25$, the recognition rate of net (1) is the highest, reaching 92.39\%. The recognition rates of three BP networks are shown in Table 3 under their respective optimal weight coefficients.

Table 2 The recognition rates of net(1) under different weight coefficients

\begin{tabular}{|c|c|c|c|}
\hline $\begin{array}{c}\text { The group of } \\
\text { experiments }\end{array}$ & $w_{1}$ & $w_{2}$ & $\begin{array}{c}\text { Recognition } \\
\text { rate }\end{array}$ \\
\hline 1 & 1 & 0 & $89.67 \%$ \\
\hline 2 & 0.875 & 0.125 & $91.3 \%$ \\
\hline 3 & 0.75 & 0.25 & $92.39 \%$ \\
\hline 4 & 0.625 & 0.375 & $90.76 \%$ \\
\hline 5 & 0.5 & 0.5 & $88.59 \%$ \\
\hline 6 & 0.375 & 0.625 & $87.5 \%$ \\
\hline 7 & 0.25 & 0.75 & $86.96 \%$ \\
\hline 8 & 0.125 & 0.875 & $85.87 \%$ \\
\hline 9 & 0 & 1 & $84.24 \%$ \\
\hline
\end{tabular}


Table 3 The recognition rates of three networks under respective optimal weight coefficients

\begin{tabular}{|c|c|c|c|}
\hline network & $\mathrm{w}_{1}$ & $\mathrm{w}_{2}$ & recognition rate \\
\hline $\operatorname{net}(-1)$ & 0.5 & 0.5 & $100 \%$ \\
\hline $\operatorname{net}(0)$ & 0.625 & 0.375 & $95 \%$ \\
\hline $\operatorname{net}(1)$ & 0.75 & 0.25 & $92.39 \%$ \\
\hline
\end{tabular}

The recognition results of partial samples of three BP networks are shown in Table 4 under respective optimal weight coefficients.

The dimension of combined features of each sample is 80 , among which 1 to 32 are grid feature and the rest are the projection feature. Two features are multiplied under respective optimal weight coefficients of each network to obtain the final combined features. Namely, the input of net(-1) is

$$
V_{\text {comb }}=\left[0.5 X_{1 \times 32}, 0.5 Y_{1 \times 48}\right]
$$

the input of net $(0)$ is

$$
V_{\text {comb }}=\left[0.625 X_{1 \times 32}, 0.375 Y_{1 \times 48}\right]
$$

the input of net(1) is

$$
V_{\text {comb }}=\left[0.75 X_{1 \times 32}, 0.25 Y_{1 \times 48}\right]
$$

The ideal output of the network corresponds to the binary code of the identified sample. The actual output of the network close to 0.1 is regarded as 0.1 , and close to 0.9 as 0.9 .

Such as the sample of "A" in Table 4, the output of net(-1) is $(0.0415,0.8424,0.9566,0.0769,0.0947,0.0531)$, which is $(0.1,0.9,0.9,0.1,0.1,0.1)$ after processing of error, corresponding to the binary code (011000) of "O". Since the character "O" does not belong to the category of net(-1), the recognition of net(-1) is wrong. The output of $\operatorname{net}(0)$ is $(0.0110,-0.0293,0.8825,0.3238,0.8634,0.0954)$, which is $(0.1,0.1,0.9,0.1,0.9,0.1)$ after processing of error, corresponding to the binary code (001010) of “A”. Since the character " $A$ " belongs to the category of net $(0)$, the recognition of net(0) is right. The output of net(1) is $(0.3315,0.4640,0.0052,0.0081,0.1572,0.0934)$, which is $(0.1,0.1,0.1,0.1,0.1,0.1)$ after processing of error, corresponding to the binary code (000000) of " 0 ”. Since the character " 0 " does not belong to the category of net(1), the recognition of net(1) is wrong. Only the recognition of net(0) is correct among three networks, so

\begin{tabular}{|c|c|c|c|c|c|}
\hline \multirow{2}{*}{$\begin{array}{l}\text { Identifying } \\
\text { characters }\end{array}$} & \multirow[t]{2}{*}{ Combined Features } & \multicolumn{3}{|c|}{ Actual Outputs } & \multirow{2}{*}{$\begin{array}{l}\text { Perfect } \\
\text { outputs }\end{array}$} \\
\hline & & net(-1) & net(0) & net(1) & \\
\hline \multirow{6}{*}{8} & 61010615001515101321610282148 & 0.0638 & 0.3916 & 0.0461 & 0.1 \\
\hline & 1501151500152101028888688887 & 0.2080 & 0.5049 & 0.9938 & 0.1 \\
\hline & 7777887799788888864 & 0.8682 & 0.2577 & 0.1909 & 0.9 \\
\hline & 488122020261588881010172620 & 0.0455 & 0.9383 & 0.1284 & 0.1 \\
\hline & 2010 & 0.2429 & 0.2109 & 0.8792 & 0.1 \\
\hline & & 0.1781 & 0.4529 & 0.1065 & 0.1 \\
\hline \multirow{6}{*}{$\mathrm{A}$} & 01650016804161604111318810412 & 0.0415 & 0.0110 & 0.3315 & 0.1 \\
\hline & 121210148812150015555666 & 0.8424 & -0.0293 & 0.4640 & 0.1 \\
\hline & 6699999767778881014141414778 & 0.9566 & 0.8825 & 0.0052 & 0.9 \\
\hline & 88661414232626181717191818 & 0.0769 & 0.3238 & 0.0081 & 0.1 \\
\hline & 1611114 & 0.0947 & 0.8634 & 0.1572 & 0.9 \\
\hline & & 0.0531 & 0.0954 & 0.0934 & 0.1 \\
\hline \multirow{6}{*}{$\mathrm{C}$} & 0101012940912002160001600013 & 0.0516 & 0.1471 & 0.1075 & 0.1 \\
\hline & 00011305010849977755553 & 0.0888 & 0.3271 & 0.0904 & 0.1 \\
\hline & 334444444443333556556692024 & 0.9358 & 0.1064 & 0.8706 & 0.9 \\
\hline & 2410764444688124 & 0.0398 & 0.0672 & 0.9003 & 0.9 \\
\hline & & 0.0976 & 0.4942 & 0.1122 & 0.1 \\
\hline & & 0.0334 & 0.3779 & 0.0636 & 0.1 \\
\hline \multirow{2}{*}{3} & 29941025113148015600613700 & 0.0405 & 0.2863 & 0.1970 & 0.1 \\
\hline & 101022839924497777754433324 & 0.5656 & 0.0527 & 0.1719 & 0.1 \\
\hline
\end{tabular}
the output of net( 0$)$ is the final correct recognition result.

Table 4 The recognition results of partial samples

Co-published by Atlantis Press and Taylor \& Francis

Copyright: the authors 


\begin{tabular}{|l|l|l|l|l|l|}
\hline & 455543375593312441813137863 & 0.8559 & 0.0364 & 0.6225 & 0.1 \\
& 379212020139 & 0.0431 & 0.7305 & -0.4617 & 0.1 \\
& & 0.4392 & 0.0008 & 0.3054 & 0.9 \\
\hline \multirow{2}{*}{$\mathbf{J}$} & 0031000412004120011200013600 & 0.1595 & 0.3643 & 0.1795 & 0.1 \\
& 1614231251615514444444444443 & 0.8225 & 0.0215 & 0.8737 & 0.9 \\
& 333334447778881410107477764 & 0.7884 & 0.1052 & 0.1032 & 0.1 \\
& 44444183128285 & 0.0338 & 0.7286 & -0.2470 & 0.1 \\
& & 0.6190 & 0.0138 & 0.9365 & 0.9 \\
& & 0.1318 & 0.2288 & 0.7597 & 0.9 \\
\hline
\end{tabular}

The situation where all the three networks' recognition results are correct is shown in Table 4. Take the sample of " $\mathrm{C}$ " as an instance, and the output of net(-1) corresponds to the binary code (001000) of "8", so the recognition of net(-1) is right. The output of net( 0$)$ corresponds to the binary code (000000) of " 0 ", so the recognition of net( 0$)$ is right. The output of net(1) corresponds to the binary code (001100) of "C", so the recognition of net(1) is right. Since the recognition results of three networks are all correct, the result of each network cannot determine the final recognition result, so the Euler number of the character should be used for further recognition. Because the Euler number of "C" is 1 , the output of net(1) is the correct recognition result.

There is the situation that the recognition results of three networks are all wrong in Table 4. Such as the sample of "3", the output of net(-1) corresponds to the binary code (011000) of "O", so the recognition of net(-1) is wrong. The output of net( 0 ) corresponds to the binary code (000101) of " 5 ", so the recognition of net( 0 ) is wrong. The output of net(1) corresponds to the binary code (001001) of "9", so the recognition of net(1) is wrong, either. Because the recognition results of three networks are all wrong, the sample cannot be identified.

In summary, the proposed method of classification BP network and combined features can satisfy the identification requirements of alphanumeric characters. Using the test samples obtained in Section 6.1, the overall recognition rate of the experiments for 36 characters reached $93.2 \%$. The recognition rate of net(-1) reached $100 \%$, and that of net(0) and net(1) reached $95 \%$ and $92.39 \%$, respectively. Illegible sample, discontinuous break handwriting (such as the sample of " 3 " in Table 4) and similar characters (such as, " 0 " and "O", " 1 " and "I", "2" and " $Z$ " etc.) are the key factors causing the decline of the recognition rate. How to deal with these issues will be one of the directions for future research.

The recognition rate of the proposed method relies on the font type and resolution of characters, which is shown in Table 5. From Table 5, it can be drawn that the recognition rate of bold font with high resolution is better, which reaches $94.4 \%$.

Table 5. Recognition rates of printed characters in different font types and resolutions

\begin{tabular}{lll}
\hline \multicolumn{2}{l}{ Font type Resolution (Dpi) } & $\begin{array}{l}\text { Recognition } \\
\text { rates \% }\end{array}$ \\
\hline normal & $75-100$ & 87.41 \\
& $150-200$ & 93.60 \\
italic & $75-100$ & 86.61 \\
& $150-200$ & 92.31 \\
bold & $75-100$ & 91.70 \\
& $150-200$ & 94.40 \\
italic bold 75-100 & 90.52 \\
& $150-200$ & 93.30 \\
\hline
\end{tabular}

In order to show the effectiveness of the proposed method, we use some existing methods to recognize the samples characters in Section 6.1, and the results are shown in Table 6, where we choose 400 training samples and 150 test samples. Results on lines marked by a '*' have been previously published and are given for comparison purposes. The first compared method is the way of choosing different BP network to recognize a character according to its Euler number (EBP). It shows that EBP performs the worst recognition rate in all methods due to the misjudgment of Euler number leading to a wrong classification of BP network. The recognition rate of HMM is less than $90 \%$, although a bit better than EBP, and this mainly comes from the limitation of its single machine learning mechanism. The way of combining neural gas and learning vector quantization contributes CAM to having a recognition rate more than $90 \%$. The proposed methodology has a best recognition rate, more than $93 \%$, due to the fact that we have synthetically considered combing features and BP network classification. 
Table 6. The compared results of different methods

\begin{tabular}{llll}
\hline & $\begin{array}{c}\text { No. of train } \\
\text { patterns }\end{array}$ & $\begin{array}{c}\text { No. of test } \\
\text { Patterns }\end{array}$ & $\begin{array}{l}\text { Recognition } \\
\text { rates \% }\end{array}$ \\
\hline *EBP & 400 & 150 & 80.3 \\
*HMM [4] & 400 & 150 & 88.4 \\
*CAM [9] & 400 & 150 & 90.6 \\
$\begin{array}{l}\text { Proposed } \\
\text { methodology }\end{array}$ & 400 & 150 & 93.2 \\
\hline
\end{tabular}

\section{Conclusions}

This paper achieved the recognition of alphanumeric characters based on BP network classification and the combined features. Firstly, we carry on a coarse classification using the structural feature of Euler number and establish one BP network for each category, with the combination of grid feature and projection feature as the input of each BP network. If an object is to be identified, its combined features will be simultaneously imported into the three networks. The final recognition result is determined by comprehensive analysis of the outputs of three networks. Experimental results showed that the coarse classification can improve the recognition ability and efficiency of BP network. From the experiment, this paper has gotten the optimal weight coefficients of combined features for all three BP networks, which can further improve the recognition rate. This method can be embedded into the system of library call number or license plate, and it can be used in handwriting character recognition. So it has good practical values.

\section{Acknowledgments}

The paper has been partially supported by the Specialized Research Fund for the Doctoral Program of Higher Education of China (20114101110005). The authors would like to thank the editors and anonymous referees for their constructive comments and suggestions that led to an improved version of this paper.

\section{References}

1. S.F. Ding, W.K. Jia and C.Y. Su, Research of pattern feature extraction and selection, in Proceedings of the 7th International Conference on Machine Learning and Cybernetics, (Kunming, China, 2008), pp. 466-471.

2. S. Günter and H. Bunke, Feature selection algorithms for the generation of multiple classifier systems and their application to handwritten word recognition, Pattern Recognition Letters, 25(11) (2004) 1323-1336.

3. G. E. Bandara, S.D. Pathirana and P.M. Ranawana, Use of fuzzy feature descriptions to recognize handwritten alphanumeric characters, In Proc. of IEEE International Conference on Fuzzy Systems, (Singapore, 2002), pp.1586-1591.

4. R. Plamondon and S.N. Srihari. On-line and off-line handwriting recognition: A comprehensive survey, IEEE
Transactions on Pattern Analysis and Machine Intelligence, 22(1) (2000) 63-84.

5. K.I. Kim, K. Jung, S.H. Park and H.J. Kim, Support vector machines for texture classification, IEEE Transactions on Pattern Analysis and Machine Intelligence, 24(11) (2002) 1542-1550.

6. N.C.Woods, O.B.Longe, A.B.C.Roberts, A Sobel Edge Detection Algorithm Based System for Analyzing and Classifying Image Based Spam, Journal of Emerging Trends in Computing and Information Sciences, 4(3) (2012) 506-511.

7. D. Keysers, W. Mcherey and H. Ney, Adaptation in statistical pattern recognition using tangent vectors, IEEE Transactions on Pattern Analysis and Machine Intelligence, 26(2) (2004) 269-274.

8. H. Nemmour and Y. Chibani, Handwritten digit recognition based on a Neural-SVM combination, International journal of computers and applications, 32(1) (2010) 104-109.

9. F. Camastra and A. Vinciarelli, Combining neural gas and learning vector quantization for cursive character recognition, Neurocomputing, 51 (2003) 147-159.

10. L. Heutte, T. Paquet, J.V. Moreau, Y. Lecourtier and C. Olivier, A structural/statistical feature based vector for handwritten character recognition, Pattern Recognition Letters, 19(7) (1998) 629-641.

11. C.L. Liu, K. Nakashima, H. Sako and H. Fujisawa, Handwritten digit recognition: benchmarking of state-of-the-art techniques, Pattern Recognition, 36 (2003) 2271-2285.

12. J.H. Cai and Z.Q. Liu, Integration of structural and statistical information for unconstrained handwritten numeral recognition, IEEE Transactions on Pattern Analysis and Machine Intelligence, 21(3) (1999) 263-270.

13. N. Thome and A. Vacavant, A combined statistical-structural strategy for alphanumeric recognition. Lecture Notes in Computer Science, 4842(2) (2007) 529-538.

14. X.B. Lu, X.J. Ling and B. Liu, License plate character recognition based on combination feature, Chinese Journal of Scientific Instrument, 27(7) (2006) 698-701.

15. Z.-C. Li and C.Y. Suen, Partition-combination method for recognition of handwritten characters, Pattern Recognition Letters, 21(8) (2000) 701-720.

16. G. Vamvakas, B. Gatos and S.J. Perantonis, Handwritten character recognition through two-stage foreground sub-sampling. Pattern Recognition, $43 \quad$ (2010) 2807-2816.

17. O. Pauplin and J. M. Jiang, DBN-based structural learning and optimisation for automated handwritten character recognition, Pattern Recognition Letters, 33 (2012) 685-692.

18. Z.X. Ge and Z.Q. Sun, Neural Network Theory and MATLAB R2007 Implementation (Electronics Industry Press, Beijing, 2008). 